\title{
New Study of Stability for New Lorenz-like System
}

\author{
Ahmmed M. Juma'a \\ College of Computer Sciences and Mathematics \\ University of Mosul
}

Received on: 27/09/2011

\author{
Ala'a A. Hammodat \\ hammodat2002@yahoo.com \\ College of Education \\ University of Mosul
}

Accepted on: 14/12/2011

\begin{abstract}
In this paper, we studied differential system like of three dimensional Lorenz system. Nonlinear characteristic and basic dynamic properties of three dimensional autonomous system are studied by means of nonlinear dynamics theory, including the stability and we found that the value $b=-1 / 4$ is effected by the form of the roots.

Keywords: New Lorenz-like system, stability, Routh-Hurwitz.

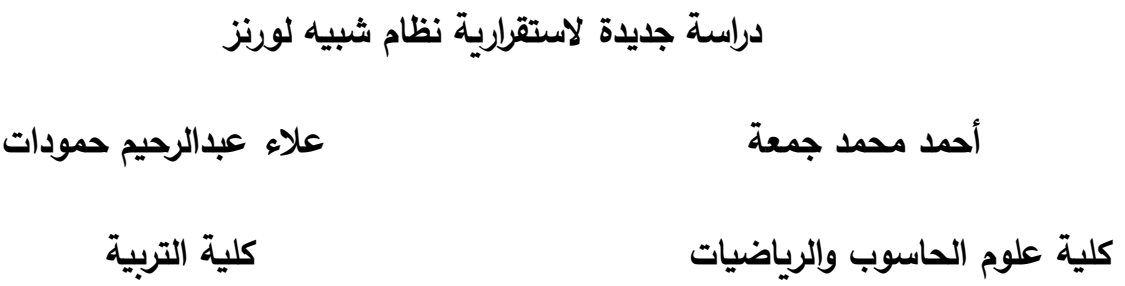

جامعة الموصل

تاريخ القبول : 2011/12/14

2011/09/27 : تاريخ الاستلام

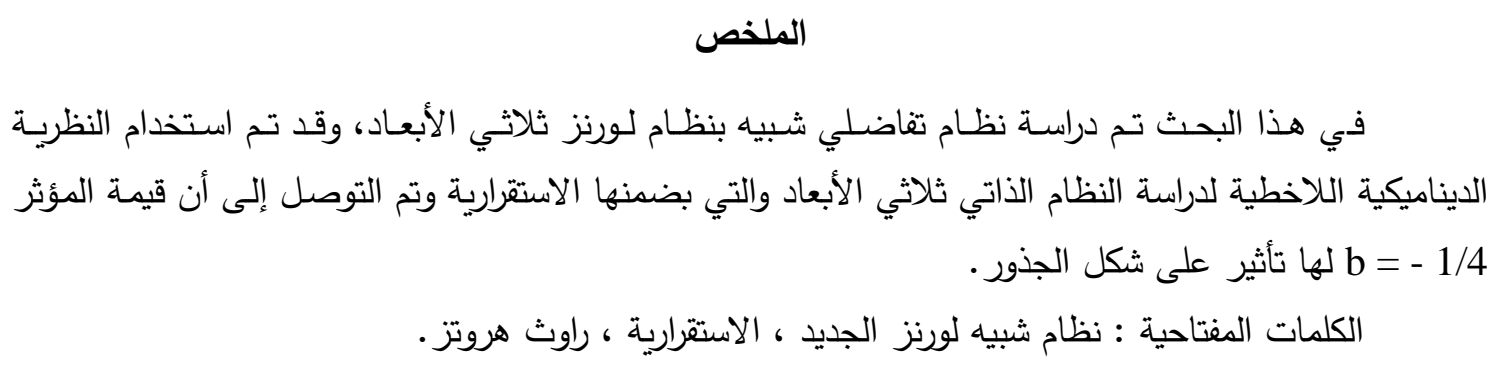

\section{1- Introduction:}

Nonlinear dynamics, commonly called the chaos theory, changes the scientific way of looking at the dynamics of natural and social systems, which has been intensively studied over the past two decades . Chaos is very interesting phenomenon closely related to nonlinear systems, which accurse so frequently that it has become important for workers in many disciplines to have a good grasp of the fundamental and basic tools of the science of chaotic dynamics [4].

All this work stems ultimately from the original investigation of an extraordinary three - dimensional nonlinear system by the mathematical meteorologist Lorenz. In 1963, he discovered chaos in a simple system of three autonomous ordinary differential equations in order to describe the simplified Rayleigh - Bernard problem 
$[5,6]$. It's notable that the Lorenz system has seven terms on the right - hand side , two of which are nonlinear $(x y, x z)$. [5]

In 1999 , Chen found a similar, but nonequivalent chaotic attractor [3], which is now known to the dual of the Lorenz system, in a sense defined [2]: when expressing the system in linear and nonlinear where the linear part has the constant matrix $A=\left[a_{i j}\right]_{3 \times 3}$, the Lorenz system satisfies the condition $a_{12} a_{21}>0$, while the Chen system satisfies $a_{12} a_{21}<0$. In 2002, Lü and Chen reported a new chaotic system called Lü system [8], which satisfies the condition $a_{12} a_{21}=0$, and bridges the gap between the Lorenz and Chen systems [5].

At the same year, a unified chaotic system was created that connects Chen chaotic system to the Lorenz chaotic system through the Lü chaotic system [7]. Last year $\mathrm{Li}$, et al [5] presented a new Lorenz - like system where the sign of the crucial condition $a_{12} a_{21}$ is only determined by parameter $\mathrm{b}$ [9].

\section{2- Dynamical Behavior of the New Chaotic Attractor:}

New Lorenz- like system, it's mathematical model that is a system of nonlinear ordinary differential equations which has the following form:

$$
\begin{aligned}
& d x / d t=a(y-x) \\
& d y / d t=a b x-a x z \\
& d z / d t=x y-c z
\end{aligned}
$$

where $(x, y, z)^{T} \in R^{3}$ is the state variables of the system. a, b and $\mathrm{c}$ are parameters . There are six terms on the right-hand side, but only relies on two quadratic nonlinearities xy and xz. [5]

The system (1) would satisfy one of the following cases via the crucial condition set by Vanecek and Celikovsky [10], $a_{12} a_{21}<0$, when $b<0, a_{12} a_{21}>0$, when $b>0$, and $a_{12} a_{21}=0$, when $b=0$. This shows that the sign of the crucial condition $a_{12} a_{21}$ is determined only by parameter $b[9]$.

The following briefly describe some basic properties of the system (1)

\section{1- Symmetry and Invariance:}

First, note that the system (1) has a symmetry $S$ because the transformation:

$S:(x, y, z) \rightarrow(-x,-y, z)$

Which permits system invariant for all values of the system parameters $a, b$ and $c$. Obviously, the $\mathrm{z}-$ axis itself is an orbit, that is if $x=0, y=0$ at $t=0$ then $x=0, y=0$ for all $t>0$. Moreover, the orbit on $z$-axis tends to the origin as $t \rightarrow \infty$. And the transformation $S$ indicates that the system is symmetrical on the $z$-axis, for instance, if $\varphi$ is the solution of the system, and $S \varphi$ is too. [5]

\section{2- Dissipative:}

The system (1) can be a dissipative system, because the divergence of the vector field, also called the trace of the Jacobian matrix is negative if and only if the sum of the parameters a and $\mathrm{c}$ is positive, that is $a+c>0$, 


$$
\begin{aligned}
& \operatorname{div} \vec{V}=\frac{\partial \dot{x}}{\partial x}+\frac{\partial \dot{y}}{\partial y}+\frac{\partial \dot{z}}{\partial z}=\operatorname{Tr}(J)=-(a+c) \\
& V(t)=V(0) e^{-(a+c) t}
\end{aligned}
$$

So, the system will always be dissipative if and only if when $a+c>0$, with an exponential: $\frac{d \vec{V}}{d t}=e^{-(a+c)}$, see [5].

\section{3- Critical Points:}

The critical points of the system (1) can be easily found by solving the three equations $\dot{x}=\dot{y}=\dot{z}=0$, which lead to

$$
\begin{aligned}
& a(y-x)=0 \\
& a b x-a x z=0 \\
& x y-c z=0
\end{aligned}
$$

It can be easily verified that there are three critical points $P_{0}(0,0,0), P_{\mp}(\mp \sqrt{b c}, \mp \sqrt{b c}, b)$

If $b c>0$, but if $b c<0$, there is only one critical point $P_{0}(0,0,0)$; as for $b c=0$, there is only one critical point but which dependent on the value of the parameter $b$, which is denoted as $(0,0, b), b \in R$. [5]

In [5], this system is studied when the parameter $b c>0$. In [9], shows that this system at the origin is asymptotically stable when $b<0$, and unstable when $b>0$, but without proof

In this paper we studied the same system when the parameter $b<0$ and $b=0$, and we found when $b<0$ that the value $b=-1 / 4$ is affected on the form of the roots.
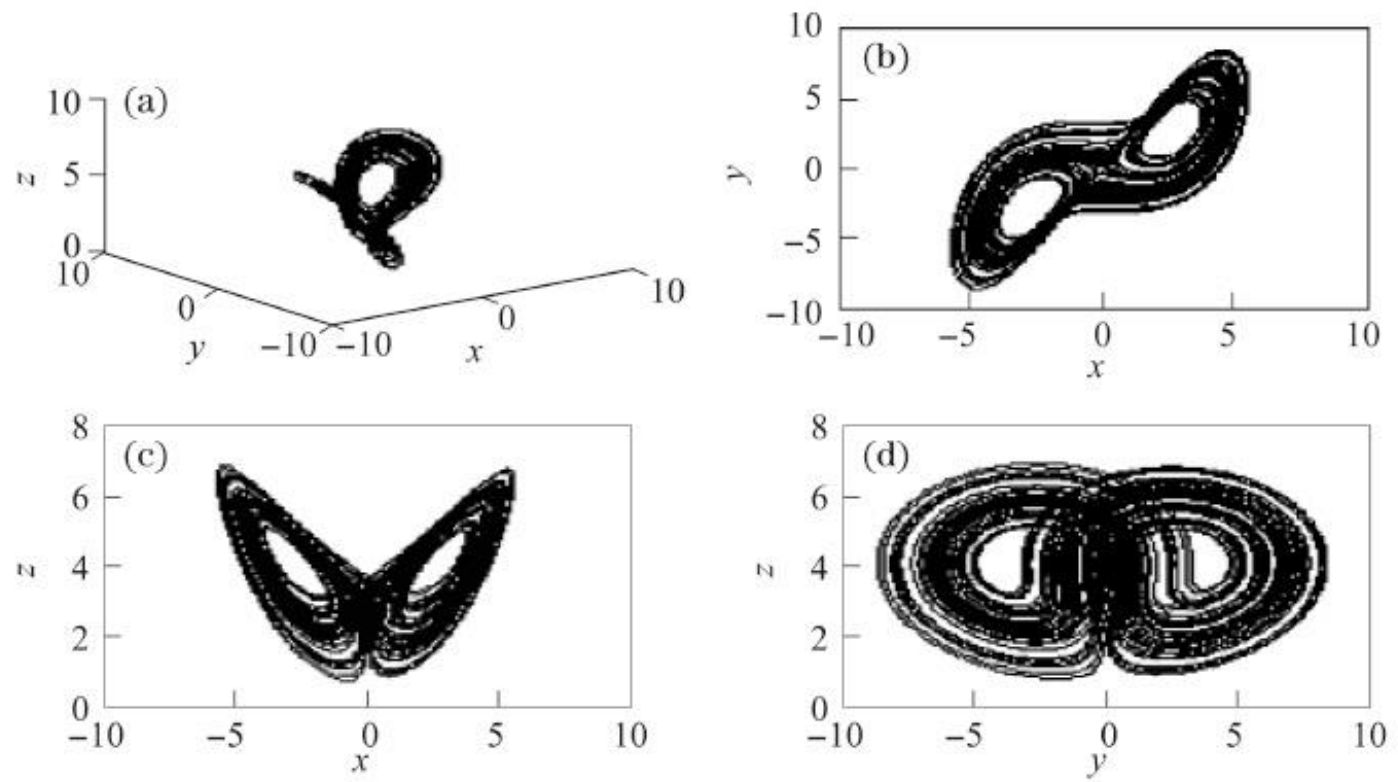

The Chaotic Attractor of the New Lorenz-like System (1)

\section{3- Helping Results:}

\section{Remark 1 (Routh-Hurwitz Test) [1]:}

All roots of the indicated polynomial have negative real parts precisely when the given conditions are met : 


$$
\lambda^{3}+a \lambda^{2}+b \lambda+c: a>0, c>0, a b-c>0
$$

Remark 2 (Critical Cases) [1]:

Critical cases in the theory of stability for differential equation means, that cases when the real part of all roots of the characteristic equation has no positive with the real part of at least one root being zero, other express which is neither stable nor unstable.

Remark 3 [5]:

The characteristic polynomial of system (1) at the critical point $P_{0}$ is :

$$
f(\lambda)=\lambda^{3}+(a+c) \lambda^{2}+\left(a c-a^{2} b\right) \lambda-a^{2} b c=0
$$

\section{Theorem 1 [5]:}

The solution of system (1) at the critical point $P_{0}=(0,0,0)$ is always unstable for $a \neq 0, b>0$.

Now, we only need to consider the stability of system (1) at $P_{+}(\sqrt{b c}, \sqrt{b c}, b)$, because the system (1) is invariant under the transformation $(x, y, z) \rightarrow(-x,-y, z)$

Under the linear transformation $(x, y, z) \rightarrow(X, Y, Z)$

$$
\begin{aligned}
x & =X+\sqrt{b c} \\
T=\quad y & =y+\sqrt{b c} \\
z & =Z+b
\end{aligned}
$$

After substituting (6) in (1) we get :

$$
\begin{aligned}
& \dot{X}=a(Y-X) \\
& \dot{Y}=-a X Z-a \sqrt{b c} Z \\
& \dot{Z}=X Y+\sqrt{b c}(X+Y)-c Z
\end{aligned}
$$

\section{Remark 4 [5]:}

The characteristic polynomial of system (7) at the critical point $P_{0}^{\prime}(0,0,0)$ is:

$$
f(\lambda)=\lambda^{3}+(a+c) \lambda^{2}+(a c+a b c) \lambda+2 a^{2} b c=0
$$

\section{Theorem 2 [5]:}

The solution of system (7) at the critical point $P_{0}^{\prime}(0,0,0)$ is asymptotically stable if and only if

$$
a+c>0,(a+c)(a c+a b c)>2 a^{2} b c
$$

It's easy to verify that the equation (8) has a pair of conjugate purely imaginary eigenvalues and one real negative eigenvalues if and only if the conditions are satisfied as follows:[5]

$$
\begin{aligned}
& a+c>0, \\
& a c(b+1)>0, \\
& a+c+b c=a b,
\end{aligned}
$$

\section{Remark 5 (Critical Value)[5]:}


New Lorenz - like system has critical value $b=0$ at the origin and $c=\frac{a(b-1)}{(b+1)}$ at the critical point $P_{+}$.

\section{4 - Main Results:}

Theorem 3: In system (1),

1 - If $b<0$, and $a, c>0$, the critical point $P_{0}=(0,0,0)$ is asymptotically stable.

2- If $b=0$, then the critical point $P_{0}=(0,0,0)$ is a critical case.

Proof: Using Routh -Hurwitz criterion, the equation (5), has all roots with negative real parts if and only if the conditions are satisfied as follows:

$$
\begin{aligned}
& A>0, \\
& C>0, \\
& A B>C,
\end{aligned}
$$

Since $A=a+c, B=\left(a c-a^{2} b\right)$ and $C=\left(-a^{2} b c\right)$, where $a, c$ are positive parameters, and $b<0$. Consequently, $A>0$ always and $C>0$ also, we must prove that $A B>C$ therefore, $a c+c^{2}-a^{2} b>0$, the proof of the first condition is complete. While if $b=0$, then satisfied remark 2 , hence the system (1) is critical case, the proof is complete.

Theorem $4:$ In (5), the relation between the parameter $b$ and the roots when $b<0$, has the following cases:

1- If $b \in(-\infty,-1 / 4)$, we get $\lambda_{1}=-c$ and $\lambda_{2,3}=-k \mp i m$

2- If $b=-1 / 4$, we get $\lambda_{1}=-c$ and $\lambda_{2}=\lambda_{3}=-k$

3 - If $b \in(-1 / 4,0)$, we get $\lambda_{1}=-c$ and $\lambda_{2}=-k_{1}, \lambda_{3}=-k_{2}$,

where $k, k_{1}, k_{2}$ and $\mathrm{m}$ are positive constant.

Proof: By analysis equation (5) we get

$$
-(c+\lambda)\left(\lambda^{2}+a \lambda-a^{2} b\right)=0
$$

and hence $\lambda_{1}=-c$ and $\lambda_{2,3}=\frac{-a+a \sqrt{1+4 b}}{2}$, therefore if $b \in(-\infty,-1 / 4)$, we get $\lambda_{1}=-c$ and $\lambda_{2,3}=-k \mp i m$, while if $b=-1 / 4$, we get $\lambda_{1}=-c$ and $\lambda_{2}=\lambda_{3}=-k$, finally if $b \in(-1 / 4,0)$, we get $\lambda_{1}=-c$ and $\lambda_{2}=-k_{1}, \lambda_{3}=-k_{2}$

The proof is complete.

Theorem 5: In system (7)

1- if $c>\frac{a(b-1)}{(b+1)}, a, b>0$, then the critical points $p_{\mp}$ are all asymptotically stable.

2- If $c<\frac{a(b-1)}{(b+1)}, a . b>0$, then the critical points $p_{\mp}$ are all unstable.

3- If $c=\frac{a(b-1)}{(b+1)}, a, b>0$, then the system (7) is a critical case.

Proof : Using Routh - Hurwitz criterion, the equation (8) has all roots with negative real parts if and only if the condition are satisfied as follows: 


$$
\begin{aligned}
& A>0 \\
& C>0 \\
& A B>C
\end{aligned}
$$

Since, $A=a+c, B=(a c+a b c)$ and $C=2 a^{2} b c$, where $a, b, c$ are positive parameters. Consequently, $A>0$ always and $C>0$, also we must proof that $A B>C$, therefore $(a+c) a c(1+b)>2 a^{2} b c \Rightarrow c>\frac{a(b-1)}{(b+1)}$, the proof of the first condition is complete.

While, if $c<\frac{a(b-1)}{(b+1)}$, then one of Routh- Hurwitz conditions is not satisfied. Consequently, the system (7) is unstable. Finally, if $c=\frac{a(b-1)}{(b+1)}$, then satisfied remark 2 , hence the system (7) is critical case, the proof is complete.

\section{5- Illustrative Examples:}

Example 1: Investigation for stability of system (1) at the critical point $P_{0}(0,0,0)$

$$
\begin{aligned}
& \dot{x}=5(y-x) \\
& \dot{y}=-20 x-5 x z \\
& \dot{z}=x y-2 z
\end{aligned}
$$

where $a=5, b=-4, c=2$

Solution: from equation (5) we get $f(\lambda)=\lambda^{3}+7 \lambda^{2}+110 \lambda+200=0$

And by using Routh - Hurwitz conditions we get $a_{1}=7>0, a_{3}=200>0$ and $a_{1} a_{2}-a_{3}=570>0$, and hence, the first part of theorem(3) is hold, therefore the system is asymptotically stable. While if $a=5, b=0, c=2$, then the system (1) is a critical case, and the second part of theorem(3) is hold.

Example 2: Investigation for stability of system (1) at the critical points $P_{\mp}(\mp \sqrt{b c}, \mp \sqrt{b c}, b)$ where $a=5, b=4, c=4$

Solution: from equation (8) we get : $f(\lambda)=\lambda^{3}+9 \lambda^{2}+100 \lambda+800=0$, and by using Routh-Hurwitz conditions, we get $a_{1}=9>0, a_{3}=800>0$ and $a_{1} a_{2}-a_{3}=100>0$, hence the system is asymptotically stable, and the first part of theorem (5) is hold.

While if $a=5, b=4, c=2$, we get: $f(\lambda)=\lambda^{3}+7 \lambda^{2}+50 \lambda+400=0$, and by using Routh-Hurwitz conditions we get $a_{1}=7>0, a_{3}=400>0, a_{1} a_{2}-a_{3}=-50<0$, hence the system is unstable. and the second part of theorem (5) is hold.

Finally, if $c=3$, we get $a_{1}=8>0, a_{3}=600>0$ and $a_{1} a_{2}-a_{3}=0$. Hence, the system is a critical case, and third part of theorem (5) is hold.

\section{6- Conclusions:}

In this paper, we studied the stability for the New Lorenz-like system by using Routh- Hurwitz method (this method depended on the previous methods on the estimate of the signs of the roots without finding the value of these roots), and we conclude that the stability of this system depended on the parameter $b$. 


\section{REFERENCES}

[1] Borrelli R.L., Coleman C.S., "Differential Equations", New-York, John Wiley and Sons, Inc, 1998.

[2] Celikovsky S., Chen G.R., "On generalized Lorenz canonical form of chaotic systems via the nonlinear observed approach", [J], Int. J. of Bifurcation and Chaos, 2002, 8, P.P., 1789-1812.

[3] Chen G.R., Ueta. T., "Yet another chaotic attractor", [J]. Int. J . of Bifurcation and Chaos, 1999, 9, P.P. 1465-1466.

[4] Jigui J., Zhengwen T., Hui Y., "Synchronization Controlling of anew Lorenz like chaotic systems using partial states feedback", Authorized licensed use limited to IEEE Xplore, 2009.

[5] Li X-F., Chu Y-D., Xu D-L., Zhang J-G., "Nonlinear dynamics and circuit implementation of a new Lorenz - like attractor", [J]. Chaos solutions and Fractals, 2009, 45, 5, P.P. 2360-2370.

[6] Lorenz, E.N., "Deterministic nonperiodic flow", [J] . Journal of the Atmospheric Science, 1963, 20, 2, P.P. 130-141.

[7] Lü, J., "Bridge the gap between the Lorenz system and the Chen system", [J]. Int. J. of Bifurcation and Chaos, 2002, 12, 12, P.P. 2917-2926.

[8] Lü, J.H, Chen G.R., "A new chaotic attractor coined", [J]. Int, J. of Bifurcation and Chaos 2002, 12, 3, P.P.659-661.

[9] Zhang, Q., Shu, Y-L., Zhang, W-X., "Controlling and Synchronization of a new Lorenz- like chaotic system", Journal of East China, normal university (Natural Science), 2010, 1, P.P. 52-61.

[10] Vanecek A., Celikovsky S., "Control System": From linear analysis to synthesis of chaos [M]: London: prentice Hall, 1996. 